\title{
Applying Selective Liquid-Phase Deposition to Create Contact Holes in Plasma Damage-Free Process
}

\author{
Ching-Fa Yeh, and Chien-Hung Liu \\ Department of Electronic Engineering \& Institute of Electronics, \\ National Chiao-Tung University \\ 1001 Ta-Hsueh Road, Hsinchu, TAIWAN \\ Tel: 886-3-5712121 ext.54242, Fax: 886-3-5711992 \\ E-mail: u8411802@cc.nctu.edu.tw
}

\begin{abstract}
We apply an alternative plasma damagefree process -- the selective liquid-phase deposition (S-LPD), instead of the conventional RIE to form metal/semiconductor contact holes. This paper studies the performance comparison between $S$ $L P D$ and RIE to form contact hole in $n^{+/ p}$ junction diode, Schottky diode, and ohmic contact. In our experiments, if the plama-free S-LPD technique is adopted, there is excellent performance including lower reverse current, lower ideality factor, higher forward current, higher Schottky barrier, lower contact resistance and better thermal stability in these devices.
\end{abstract}

RIE is widely employed to etch silicon oxide to form contact holes owing to its anisotropic etching ability. However, because of inevitable energetic ions and fluorine-contained radicals in plasma ambient, RIE easily causes energetic surface contamination and if damage in $\mathrm{SiO}_{2} / \mathrm{Si}$ interface. In addition, the selectivity issue of RIE has become more critical in ultra-shallow junction, because overetch is less allowed. To avert these problems, an alternative method without etching is urgently required to replace conventional RIE method. According to our previous investigation ${ }^{[1],[2]}$, the liquid-phase deposition (LPD) method can selectively deposit silicon oxide against photoresist, shown in Fig. 1, if the condition of hydro-fluorosilicic acid solution is adequately controlled. This SEM photograph reveals the possibility of further applying S-LPD to the deep submicron process. In this work, we apply S-LPD to form contact holes in $\mathrm{n}^{+} / \mathrm{p}$ diodes, Schottky diodes and Kelvin resistors and, thereby, study the superiority of utilizing plasma-free S-LPD over the conventional method by comparing the current-voltage (I-V) characteristics of prepared devices.

The P-type (100) wafers with 15-25 $\Omega-\mathrm{cm}$ resistivity are adopted in the $\mathrm{n}^{+} / \mathrm{p}$ diodes and Kelvin resistors fabrication. Following formation of the channel stopper and field oxide, phosphorus with dose of $5 \times 10^{15} \mathrm{~cm}^{-2}$, and energy of $40 \mathrm{keV}$ is implanted to make $n^{+} / p$ junction. As the left side of Fig.2 indicates, for the S-LPD samples, the photoresist on the site of contact hole region is first patterned and, then, the LPD oxide is selectively grown on the region without photoresist. After removing the photoresist, contact holes without plasma damage are automatically formed. For the RIE samples, as shown in the right side of Fig.2, LPD oxide is globally deposited all over the wafers, and then the contact holes are formed by using RIE technique (only $\mathrm{CF}_{4}$ gas: $5 \mathrm{sccm}$, 50mtorr; rf power: $100 \mathrm{~W}$ ) through lithography photoresist patterns. After metallization, some $\mathrm{n}^{*} / \mathrm{p}$ junction diodes are sintered at $400^{\circ} \mathrm{C}, 30 \mathrm{~min}$, in N2. The Kelvin resistors, illustrated in Fig.3, were fabricated with the same condition. Meanwhile, for Schottky diodes, the fabrication procedures closely resemble those of $n \% / p$ diodes except for $1-5 \Omega$-cm resistivity $N$-type (100) wafers used and no ion-implantation needed. In these divices, typical current-voltage $(\mathrm{I}-\mathrm{V})$ characteristics and contact resistance are investigated to clarify the better performance of damage-free devices.

Fig.4 shows the I-V characteristics of $n^{+} / p$ diodes fabricated by S-LPD, RIE and partial-RIE, respectively. Herein, the partial RIE method is adopted to buffer some plasma damage. Such an application suggests that the $85 \%$ oxide is etched by RIE and, then, the remaining $15 \%$ oxide is etched by wet-etching in BHF solution. The $\mathrm{n}^{+} / \mathrm{p}$ junction area is $100 \times 100 \mu \mathrm{m}$ and the contact hole area is $60 \times 60 \mu \mathrm{m}$. The reverse current of S-LPD samples is much less than those of RIE and partial RIE samples. Several key characteristic parameters are summarized in Table1. Although partial-RIE can slightly improve some diode characteristics relatively to RIE only used, the damage-free S-LPD diodes exhibit excellent performance relative to the RIE or partialRIE ones whether in forward bias or in reverse bias.

Table 1 also lists the reverse area current density, $J_{R A}$, and the reverse periphery current density, JRP. To extract these two reverse current density, we have to apply two $\mathrm{n}^{+} / \mathrm{p}$ diodes with different size. 
Combining the $200 \times 200 \mu \mathrm{m}$ diode and the foregoing $100 \times 100 \mu \mathrm{m}$ diode, by the following equation:

$$
I=J_{R A} W L+J_{R p} \times 2(L+W),
$$

we can calculate the JRA and JRP. As listed in this table, S-LPD exhibits much smaller $J_{R A}$ and $J_{R P}$. Especially, the JRA of RIE is two-order higher than the JRA of S-LPD. This finding indicates that RIE damages and contaminates in contact area very seriously, relative in the periphery junction region. The damage and contamination can serve as generation centers to enlarge reverse current and serve as recombination centers to degrade ideality factor. Therefore, the performance of S-LPD diodes is much better than that of RIE diodes in reverse bias and in small forward bias because this problem doesn't exist in the S-LPD process. Meanwhile, is Fig.4 depicts, the partial RIE can reduce damage and improve reverse current. However, comparing to the S-LPD, it is still worse very much. This seems to result from the following two reasons: First, in RIE plasma ambient, there are some high energy ion or radial which can penetrate the $15 \%$ remaining oxide into the silicon bulk region. Secondly, the if radiation of RIE can also damage device. Thirdly, the micro-trenching effect ${ }^{\left[{ }^{3}\right]}$ due to ion reflection from the photoresist sidewall can enhance the etching at contact periphery and cause more serious penetration. Therefore, only using S-LPD can avoid the RIE damage problem and get the advantages due to plasma-free.

Fig.5(a) and Fig.5(b) show the diode performance before and after sintering treatment, 400 ${ }^{\circ} \mathrm{C}$, 30min, in N2. Sample diodes of aluminium directly contact to silicon are shown in Fig.5(a). After sintering treatment, the reverse current of RIE diode drastically increase. It's almost three order higher than S-LPD diode after the same sintering. Sample diodes, shown in Fig.5(b), are to add titanium silicide between aluminium and silicon. The similar trend also exists, but the increase magnitude of reverse current is not so serious. By these two figure, this results attribute to two factors as following. First, the Al spiking effect cause this degradation. Especially, the spiking effect of RIE is more serious than that of S-LPD. It indicates that RIE damage will enhance the Al spiking effect. Secondly, the contamination and impurities due to RIE further penetrate into the depletion region of $\mathrm{n}^{+} / \mathrm{p}$ junction after themal sintering and, therefore, enlarge the reverse current. Therefore, even if we add titanium silicide, the reverse current increase level of RIE is still larger than that of selective-LPD.
To investigate the Si near-surface region in ultra-shallow junction, we utilize Schottky diodes to demonstrate the plasma damage problem of nearsurface region between RIE and S-LPD. Fig.6 depicts the performance of S-LPD and RIE Schottky diodes, and Table I also summarizes the key characteristics. Before sintering, RIE sample nearly loses the rectifying characteristics of Schottky diode under reverse bias, but S-LPD sample exhibits satisfactory Schottky characteristics. However, after sintering (solid curve), the S-LPD sample still exhibits about four order smaller than that of RIE sample in reverse current; meanwhile the ideality factor 1.03 of the former is also lower than 2.03 of the latter. This finding reveals that the sintering is ni vessary for the RIE sample to release a part of $\mathrm{r}$;idues and defects from the $\mathrm{Si}$ surface, but unnecessary for the S-LPD sample. For RIE Schottky diode, the worse ideality can be attributed to the large surface recombination velocity. According to the curve slope of $\ln \{\mathrm{l} /[1-\exp (-\mathrm{qV} / \mathrm{kT})]\}$ versus forward-bias voltage, the potential barriers is $0.83 \mathrm{eV}$ and $0.6 \mathrm{eV}$ for S-LPD and RIE Schottky diode, respectively. This result attributes to that the donorlike bonding defects and the polymer residues make the depletion region thinner and the potential barrier lowering. The barrier lowering effect seriously degrades both reverse and forward I-V characteristics. Above results imply that RIE will be increasingly critical and requires more severe after-RIE-treatment in ultra-shallow junction. However, utilizing S-LPD can avert these problems, and is expected to be a highly promising candidate as novel plasma damagefree and plasma contamination-free technology.

In the contact investigation, at first, we mesure the contact resistance Rc with Kelvin Dre,istors. The Kelvin contact resistance is widely defined by the voltage 1 to 3 over the fed current 2 to 4. illustrated in Fig.3, i.e., the slope of V13-124 plot in Fi. 7. Fig.7 depicts the V-I characteristics at the curent fed from $-20 \mathrm{~mA}$ to $20 \mathrm{~mA}$ for the resistor win contact size $\mathrm{L} \times \mathrm{L}=10 \times 10 \mu \mathrm{m}^{2}$ and collar width $\mathrm{d}=10 \mathrm{im}$. The S-LPD resistor shows a linear $\mathrm{V}-\mathrm{I}$ relationship and exhibits a good ohmic contact. However, the RIE resistor shows a non-linear V-I relationship and, therefore, exhibits a non-constant and larger Rc value, especially in the small current region. This result indicates that the RIE with Thorine carbide plasma enlarges contact resistance very seriously. This serious problem seems to be resulted from that RIE causes the mobility decrease in etched surface because the impurities impinged by RIE will scatter carriers when electrons or holes move through the damaged contact surface. 
We incorporate the measured $\mathrm{Rc}$ results of different $\mathrm{L} / \mathrm{d}$ ratio into the universal curves of 4 terminals Kelvin D-resistor simulated by J. Santander et $\mathrm{a} \mathrm{l}^{[4]}$. By the measured $\mathrm{Rc} / \mathrm{Rs}$ ratio versus the contact/collar ratio L/d of resistors, shown in Fig. 8 , the $\mathrm{Rc} / \mathrm{Rs}$ values of S-LPD samples are much smaller than those of RIE samples in all resistors with various geometry size. Combining the dash lines which show the simulated universal curves in Fig.8, the $\mathrm{Lr} / \mathrm{d}$ value of S-LPD with $10 \mu \mathrm{m}$ collar is $1 / 2 \sim 1 / 8$ factor smaller to that of RIE. By this figure for the $10 \mu \mathrm{m}$ collar resistors, we estimate that the transfer length, $L_{T}=(\rho c / R s)^{1 / 2}$, of S-LPD samples is about $2.7-5.5 \mu \mathrm{m}$, but that of RIE is about $10 \sim 22 \mu \mathrm{m}$. Therefore, with $\mathrm{Rs}=26 \Omega / \mathrm{sq}$ measured, we get that the specific $\mathrm{Al} / \mathrm{Si}$ contact resistivity $\rho \mathrm{c}$, which only depends on the material and process issues, approximates to $10^{-6} \Omega-\mathrm{cm}^{2}$ for S-LPD; meanwhile, the $\rho c$ of RIE is higher than $10^{-5} \Omega-\mathrm{cm}^{2}$. Even if we cleaned the RIE contact holes with RCA cleaning in our experiments, it seems to be impossible to clean the RIE contamination, which is impinged by ifpower or dc-bias, by the conventional clean method. However, the photoresist contamination of S-LPD is easily cleaned by the conventional clean method. Because the contact resistivity is very sensitive to the clean problem in metal/Si interface ${ }^{[5]}$, the specific $\rho c$ of RIE must be much larger than that of S-LPD except that a severe and complex after-RIE-treatment is employed ${ }^{[0]}$.

In this paper, the S-LPD technique has been successfully adopted to form contact hole. As described above, the plasma-free S-LPD technique is superior to the conventional RIE with the following advantages: very low reverse current for $n+/ p$ diode, good stability for thermal treatment, rather high potential barrier for Schottky diode, and low contact resistance. In contrast, the conventional RIE technology cannot be utilized in damageless contacthole formation. The S-LPD technique is highly promising to replace conventional RIE for contact hole formation. In general, S-LPD method doesn't damage and contaminate the device surface; thus, no additional treatment is required. Therefore, the novel S-LPD technology can be applied to ultra-shallow junction and deep submicron process in the near future.

\section{Reference}

[1] C.F.Yeh, and C.L.Lin, Semicond. Sci. Tech., vol.9, p1250, 1994.

[2] C.F.Yeh, Y.C.Lee, and J.L.Su, in Proc. SPIE, vol.2879, pp260-265. 1996.

[3] T.J.Dalton. J.C.Amold, et al., I. Electrochem. Soc. vol.140, pp2395, 1993.
[4] J.Santander, M.Lozano, and C.Canes, IEEE Trans. on Elec. Dev, vol.35, no.3, pp944-950, 1993.

[5] T.Hara, and S.Kamiyama, J. Electrochem. Soc., vol.134, no.8 pp452C, 1987.

16] H.C.Tseng, C.Y.Chang, F.M.Pan, and L.P.Chen, J. Appl. Phys., vol.78, pp4710, 1995

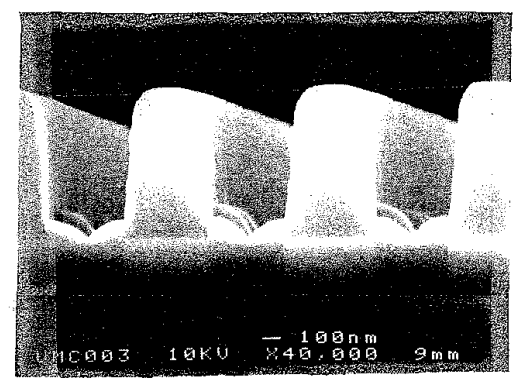

Fig.1 SEM cross section view of oxide profile against photoresist for selective-LPD deposition.

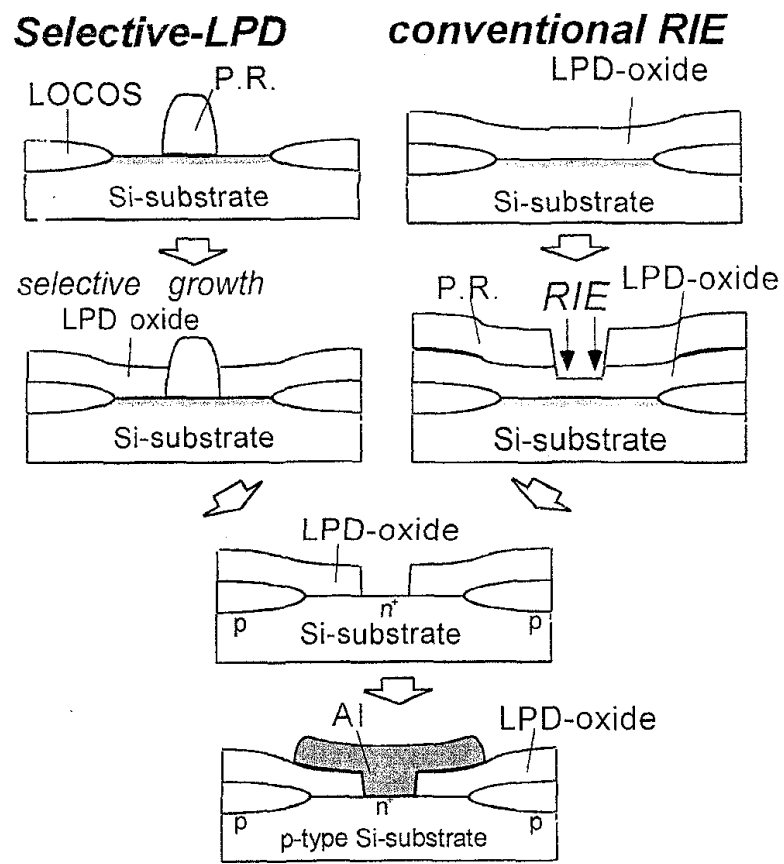

Fig.2 The brief diagram of key processes for the metal/semiconductor contact hole fabricated by the S-LPD (left) and the conventional RIE (right), respectively.
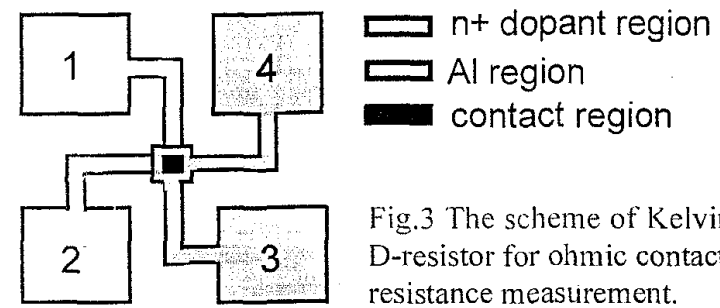

Fig.3 The scheme of Kelvin D-resistor for ohmic contact resistance measurement. 
Table 1 Key parameter comparison of $\mathrm{n}^{+} / \mathrm{p}$ diodes and Schottky diodes fabricated by S-LPD and RIE.

\begin{tabular}{|c|c|c|c|c|c|c|c|c|}
\hline & \multicolumn{5}{|c|}{$\mathrm{n}^{+} / \mathrm{p}$ junction diode } & \multicolumn{3}{|c|}{ Schottky diode } \\
\hline & \multicolumn{4}{|c|}{ before sintering } & \multirow{3}{*}{$\begin{array}{l}\text { after sintering } \\
\text { reverse current } \\
\text { (A) at } 5 \mathrm{~V}\end{array}$} & \multirow{3}{*}{$\begin{array}{l}\text { Schottky } \\
\text { ideality } \\
\text { factor } \eta \mathrm{Sc}\end{array}$} & \multirow{3}{*}{$\begin{array}{l}\text { reverse } \\
\text { current } \\
\text { (A) at }-5 \mathrm{~V}\end{array}$} & \multirow{3}{*}{$\begin{array}{l}\text { barrier } \\
\text { height } \\
(\mathrm{eV})\end{array}$} \\
\hline & \multirow{2}{*}{$\begin{array}{l}\text { ideality } \\
\text { factor } \eta\end{array}$} & \multicolumn{3}{|c|}{ reverse current at $5 \mathrm{~V}$} & & & & \\
\hline & & $\operatorname{IR}(A)$ & JRA $\left(\mathrm{A} / \mathrm{cm}^{2}\right)$ & $J_{R P}(\mathrm{~A} / \mathrm{cm})$ & & & & \\
\hline S-LPD & 1.11 & $2.77 e-11$ & $5.72 \mathrm{e}-8$ & $1.72 \mathrm{e}-9$ & $6.09 e-9$ & 1.03 & $4.14 \mathrm{e}-9$ & 0.83 \\
\hline RIE & 1.69 & $2.57 \mathrm{e}-9$ & $9.25 e-6$ & $4.10 e-8$ & $5.82 e-6$ & 2.03 & $1.85 e-5$ & 0.6 \\
\hline
\end{tabular}

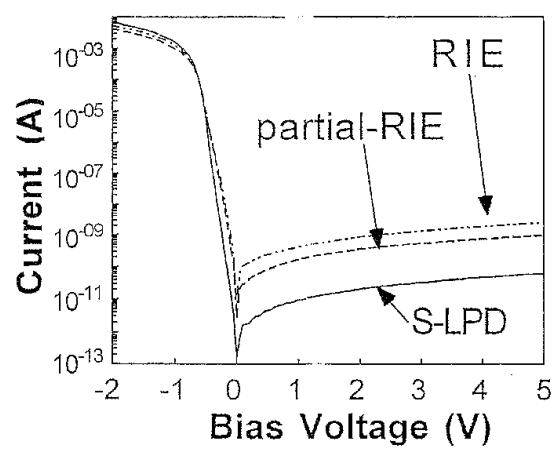

Fig. $4 \mathrm{I}-\mathrm{V}$ characteristics of $\mathrm{n}^{+} \% \mathrm{p}$ diode fabricated by S-LPD, RIE, and partial-RIE, respectively.

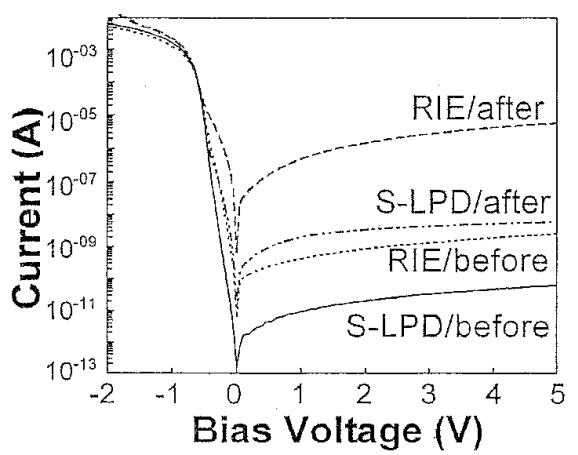

(a)

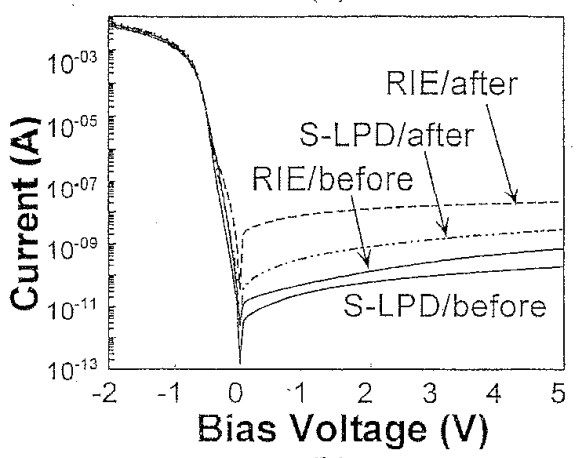

(b)

Fig.5 [-V characteristics of $n^{+} / p$ diode before/after sintering for (a) Al/Si contact, (b) Al/Ti/Si contact.

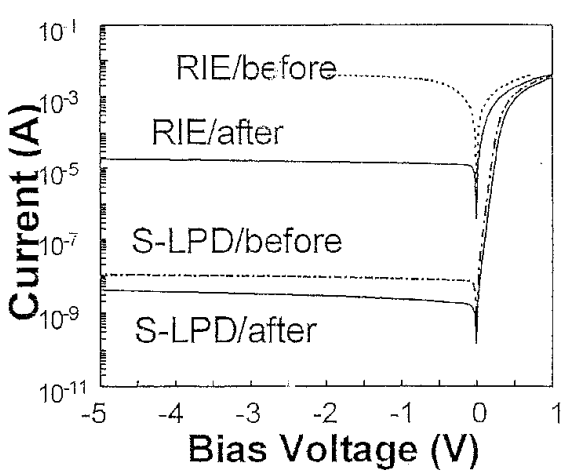

Fis.6 I-V characteristics of Schottky diodes fabricated by S-LPD and RIE, before and after sintering.

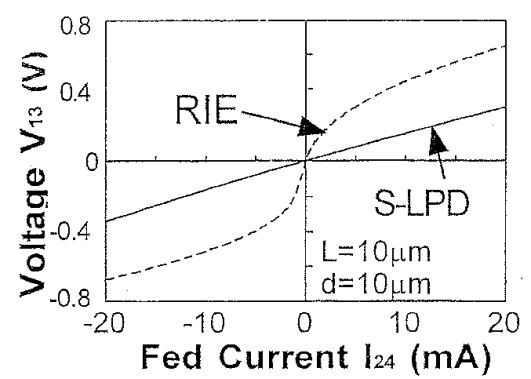

Fig.7 V-I plot of Kelvin D-resistors fabricated by S-LPD and RE, respectively.

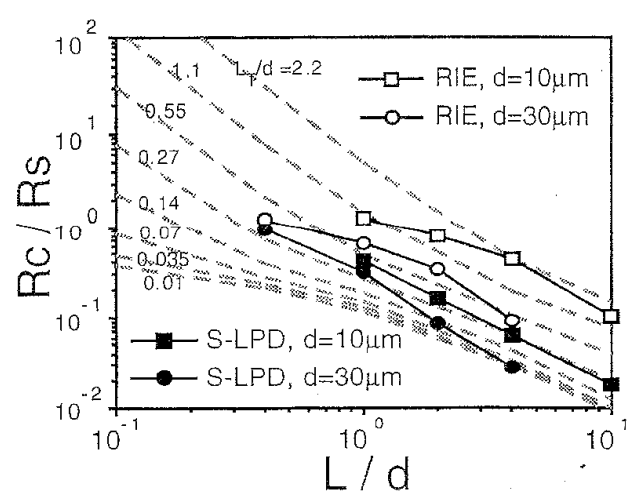

Fig. 8 Measured Rc/Rs in simulated universal curves for the Kelvin D-resistors fabricated by S-LPD and RIE, respectively. 